This method is quick and cheap; it is useful for the investigation of uncommon disease; and it allows the evaluation of several aetiological factors acting synchronously or separately. Case-control studies are now accepted by most epidemiologists as one of the methods of generating hypotheses and perhaps of testing them, but there is less agreement on whether results need validation by other methods before they become acceptable. A major criticism of case-controlled studies is that, because results tend to be achieved quickly and the topics under investigation tend to be those of public interest, public debate occurs and policy decisions are taken more rapidly than with other methods of study.

The statistical analysis of the results of case-control studies has been refined over the past 20 years, ${ }^{2-4}$ but problems remain: in particular, any retrospective study is less likely to obtain the randomness and independent selection of each individual that are found in the best prospective experimental or cohort follow-up studies. Nevertheless, as Finney ${ }^{5}$ has pointed out, the methods are there and they must be correctly used. Clearly, the choice of controls and cases is all important. Ideally the cases should be truly representative of all cases, but this may not be possible, and sometimes selection may have to be based on a single characteristic. The results can still be important when such a study is considered along with others based on different features, but if a large enough group is available it may be possible to analyse by proper stratification and obtain valid results for several entities in one groupthough there must be some suspicion of a study that whittles a population down from thousands to a handful of cases.

One of the chief problems for the investigator is the elimination of bias. Sackett ${ }^{6}$ has catalogued 35 sources of bias, and doubtless more remain to be discovered. These seem to fall into three main groups. Firstly, selection bias is the most difficult to eradicate, for it is extremely difficult to avoid differential recruitment into the case and the control groups. Patients who have been admitted to hospital present a clearly defined group, but even they are artificially selected to some extent, and one of the control groups should be drawn from patients not admitted to hospital. Another cause of selection bias is that cases may have passed through an investigation sieve that the controls have avoided. Secondly, information bias may occur more easily in a retrospective study than a prospective one: all too easily the facts collected from the records or the history may be incomplete or inaccurate. The third common source of bias is that produced by the mismatching of cases and controls-confounding factors. ${ }^{7}$ One way of minimising these is by choosing at least three independent control groups for the study.

The case-control study has had its successes, notably the original demonstration of the associations between the contraceptive pill and thromboembolism, but it has also created problems, such as the unresolved debate about the possible link between reserpine and breast cancer. Its future will depend on the care with which studies are designed, the avoidance of inappropriate use, and perhaps a better understanding of the problems of bias.

${ }^{1}$ Lane-Claypon, J E, A Further Report on Cancer of the Breast, with special reference to its associated antecedent conditions. Reports on Public Health and Medical Subjects 32: Ministry of Health. London, HMSO,

2 Cornfield, J, Fournal of the National Cancer Institute, 1950-1951, 11, 1269.

3 Dorn, H F, New England fournal of Medicine, 1959, 261, 571.

4 Mantel, N, and Haenszel, W, fournal of the National Cancer Institute, $1959,22,719$.

5 Finney, D J, fournal of Chronic Diseases, 1979, 32, 78.

${ }^{6}$ Sackett, D L, fournal of Chronic Diseases, 1979, 32, 51 .

7 Jick, H, and Vessey, M P, American fournal of Epidemiology, 1978, 107, 1.

\section{Undergraduate education in rheumatology}

Most specialties are clamouring for a share in an already overcrowded medical curriculum. Few have gathered the teachers together from all the schools in Britain to discuss what their specialty can contribute in educational terms. A recent workshop in rheumatology tried to do just that. ${ }^{1}$

The teaching of any specialty has to be seen against the background of trends in medical education. One of these is that nowadays most medical students are brighter than their teachers. The teacher may draw comfort from the axiom that a wise man may learn more from a fool than a fool can from a wise man, and that his experience may partly qualify him for the role of "master." In teaching methods the trend is away from passive learning and to active participation as the emphasis on authoritarian instruction declines. Nevertheless, every subject must be taught with an economy of effort, and, though it is a branch of general medicine, teaching in rheumatology means collaboration with clinical immunology and with orthopaedic surgery. Many clinicians still seem unaware of the other aspects of their subject that are taught elsewhere in the curriculum. The strengths and weaknesses of individual medical schools need also to be taken into account: if there is an expert in gout he will have an obvious contribution to make to the basic science teaching on purine metabolism.

The objectives of medical education may be classified as skills, knowledge, and attitudes. In rheumatology the skills include an ability to examine the musculoskeletal system (in both history taking and physical examination) and to record the findings. Too many clinicians still seem to believe that there are just four systems in the body and give scant attention to the fifth, the musculoskeletal. From a proper examination will arise the approach to differential diagnosis, and particularly the ability to identify acute problems which necessitate prompt referral for specialist advice. Chronic rheumatic diseases also provide a valuable model of the assessment of disability, and alongside that the skills in rehabilitating such patients. Moreover, rheumatologists are one group of clinicians who have to teach some of their patients to accept disability - a difficult skill which it is vital students should appreciate. Physicians also have to be aware of their own limitations, recognising that it is not necessarily in the patients' best interests to administer a multiplicity of anti-inflammatory drugs when problems arise from residual damage and not from active inflammation.

In terms of knowledge, students need to appreciate the range of rheumatic diseases, their impact in the community, their pathogenesis, and their management-the relief of pain and the treatment of other aspects of the condition. Students' enthusiasm may be roused by the breadth of the subject and the excellent results that are obtained in conditions such as gout, polymyalgia rheumatica, unilateral osteoarthrosis of the hip, and many cases of rheumatoid arthritis. Rheumatology also serves as a model for developing correct attitudes in the management of chronic disease and in the value of co-operation with members of the paramedical professions.

These reasons may help explain why so many students choose rheumatology for an elective period. Though their teachers' enthusiasm needs to take account of curriculum constraints, the subject is ideal for educational experiments. It would, for instance, be interesting to link a student, a patient, and a physician in the student's first year, with 
meetings every six months throughout the ensuing five years, to give a broader understanding of the problems and successes with such patients and the skills and attitudes needed in achieving these results.

1 Arthritis and Rheumatism Council, Workshop on Undergraduate Education in Rheumatology, Manchester, 1979.

\section{Cell surface markers in chronic lymphatic leukaemia}

Chronic lymphatic leukaemia (CLL) is the most common leukaemia seen in Western countries, though characteristically it is found in elderly patients. In about a third of cases the disease is diagnosed by accident when blood tests are done for other reasons or when splenomegaly or lymphadenopathy is detected on routine examination. Some degree of clinical and morphological overlap exists between CLL and other lymphoproliferative disorders, and the identification of immunological markers on the lymphocyte cell surface is becoming increasingly valuable in separating these diseases. Lymphocytes can be classified by their reaction with sheep erythrocytes to form E-rosettes ( $T$ cells) or by the presence of surface immunoglobulin (B cells). Other cell surface markers have already helped in subclassifying acute lymphoblastic leukaemia ${ }^{2}$ and may help to classify unusual cases of CLL. ${ }^{3}$

$\operatorname{Ig} M$ and later $\operatorname{IgD}$ appear on the lymphocyte surface in fetal life, ${ }^{4}$ so that cells possessing both surface immunoglobulins may be assumed to be more mature than those with IgM only. The cells that proliferate in CLL are monoclonal B lymphocytes that possess both $\operatorname{IgM}$ and $\operatorname{IgD}$ on their surface ${ }^{5}$ as well as complement receptors. In vitro these cells are able to synthesise immunoglobulin light chains, ${ }^{6} 7$ and occasionally patients are found to have a monoclonal immunoglobulin, usually IgM, in the serum. In these cases the serum immunoglobulin appears antigenically identical with that on the cell surface. ${ }^{8}$ Though the CLL lymphocyte is thought to be incapable of further differentiation, some patients show a picture of light-chain excretion, amyloidosis, and sometimes IgM paraproteins, suggesting a link with Waldenström's macroglobulinaemia and the mature plasma cell. ${ }^{9}$

In a few patients with CLL the lymphocytes form Erosettes, antibodies have been found against $\mathrm{T}$-cell-specific antisera, ${ }^{10-12}$ and there are no surface immunoglobulins. These patients often have small or impalpable lymph nodes but a moderate degree of hepatosplenomegaly. Leukaemic skin infiltration is usual, and the central nervous system is occasionally affected. ${ }^{12}$ The morphology of the lymphocytes is variable, but often there is a folded nucleus and copious cytoplasm containing azurophilic granules. Nevertheless, these patients do not have the mediastinal lymphadenopathy characteristic of $\mathrm{T}$-cell acute lymphoblastic leukaemia (T-ALL) and of lymphoblastic lymphoma. ${ }^{13}$ The lymphocytes do not contain the enzyme terminal transferase ${ }^{12}$ present in T-ALL cells, ${ }^{14}$ and this $\mathrm{T}$-cell variant of CLL appears to result from proliferation of a mature lymphocyte clone. ${ }^{12}$

Diffuse lymphocytic lymphomas of well-differentiated cell type (WDLL) occur predominantly in older patients and are usually widespread at diagnosis. The appearances of lymph node biopsy samples are indistinguishable from those of the tissue infiltrates of $\mathrm{CLL}^{1.5}$ and WDLL has close clinical similarities too. Immunological studies have shown that in WDLL the lymphocytes bear monoclonal B-cell markers identical with those found in B-cell CLL, ${ }^{16-19}$ and indeed WDLL is now thought to represent CLL without extension to the blood.

In the past it has been difficult to define CLL in terms of an absolute lymphocyte count because of the difficulty of separating CLL from benign lymphocytosis. Patients with total lymphocyte counts below $15 \times 10^{9} 1$ are still usually excluded..$^{29}$ Early CLL can now, however, be distinguished from benign lymphocytosis by cell surface marker studies. Patients with non-neoplastic lymphoid proliferations have a symmetrical increase in both $\mathrm{B}$ and $\mathrm{T}$ cells, whereas patients with early CLL have lymphocytes that almost exclusively show the B-cell markers of the typical disease. ${ }^{21}$

In a few patients with lymphosarcoma primitive lymphoid cells appear in the blood in the terminal stages of the disease. This clinical picture is usually easy to recognise, but the occasional patient who shows such cells early on presents diagnostic difficulties. Lymphosarcoma cells are often larger than mature lymphocytes, and contain variable amounts of clear grey-blue cytoplasm and a cleft or indented nucleus that usually has a characteristically primitive chromatin pattern. ${ }^{22}$ Considerable morphological variability exists, however, and the large amounts of immunoglobulin that are seen on the surface of lymphosarcoma cells contrast with the sparse amounts found in both CLL and WDLL. This distinction provides a reliable means of separating CLL from lymphosarcoma-cell leukaemia. ${ }^{17}$

Prolymphocytic leukaemia (PL), first described by Galton, ${ }^{23}$ is an unusual disease occurring predominantly in elderly men and occupying a position between CLL and lymphosarcomacell leukaemia. It is usually characterised by tiredness, loss of weight, sweats, fevers, massive splenomegaly, and moderate hepatic enlargement, but little or no lymphadenopathy. The blood contains many large lymphocytes with a moderate amount of cytoplasm and well-condensed nuclear chromatin with a single prominent nucleolus. Most patients have B-cell markers on the cell surfaces, ${ }^{21}$ but Catovsky and his colleagues have shown differences in surface receptors between B-cellCLL and B-cell-PL that may help differential diagnosis ${ }^{25} 26$ - principally the formation of a high proportion of rosettes in the reaction with mouse red cells in CLL, but not in PL.

Other lymphoproliferative disorders that occasionally may be confused with CLL include the Sézary syndrome and hairycell leukaemia. The Sézary syndrome was the first clearly identified $\mathrm{T}$-cell neoplasm ${ }^{27}$ and is now known to be a malignant proliferation of $\mathrm{T}$ helper cells. ${ }^{28}{ }^{29}$ Its characteristic skin lesions ${ }^{30}$ will nearly always distinguish it from CLL. On the other hand, the origin of hairy cells has been hotly debated ${ }^{26}$ and, though they are probably B cells, ${ }^{31}$ the diagnosis is made by methods other than surface marker studies. ${ }^{26}$

The identification of lymphocyte surface markers, then, is no longer the exclusive province of research workers but a practical diagnostic tool for all specialist haematology laboratories. Nevertheless, as Brouet and Seligmann ${ }^{1}$ have recently emphasised, membrane marker studies have to be interpreted with knowledge of the methodological problems and pitfalls; no single test, or even small group of tests, is likely to give an absolutely clear indication even between $\mathrm{T}$ and $\mathrm{B}$ membrane markers. Using a panel of markers is essential for accurate conclusions. We know little so far about how membrane markers evolve on neoplastic cells, but distinct changes are known to occur-for example, cell membrane markers may 